\title{
Reactive Power Optimization Avoiding Over Compensation Based On Improved Particle Swarm Optimization
}

\author{
Wang Jianfeng ${ }^{1}$, Xiang Tieyuan ${ }^{2}$ and Jin Weijian ${ }^{3}$ \\ ${ }^{1)}$ School of Electrical Engineering, Wuhan University, Wuhan, Hubei, China (xufengassy@ foxmail.com) \\ ${ }^{2)}$ School of Electrical Engineering, Wuhan University, Wuhan, Hubei, China (tyxiang@whu.edu.cn) \\ ${ }^{3)}$ College of Engineering, Northeastern University, Boston, the U.S. (jin.we@ husky.neu.edu)
}

\begin{abstract}
Improved particle swarm optimization algorithm was applied to power system reactive power optimization overcomes the traditional particle swarm optimization algorithm's problems, which are easy to fall into local optimum and with low precision. At the same time in the process of operation, this paper proposes a limit on the compensation capacity with dynamic adjustment to avoid over compensation. The proposed algorithm has been successfully applied to IEEE6 bus system, and the comparative results show that active loss and voltage levels have significant improvement.
\end{abstract}

Keywords — reactive power optimization, improved particle swarm algorithm, over compensation

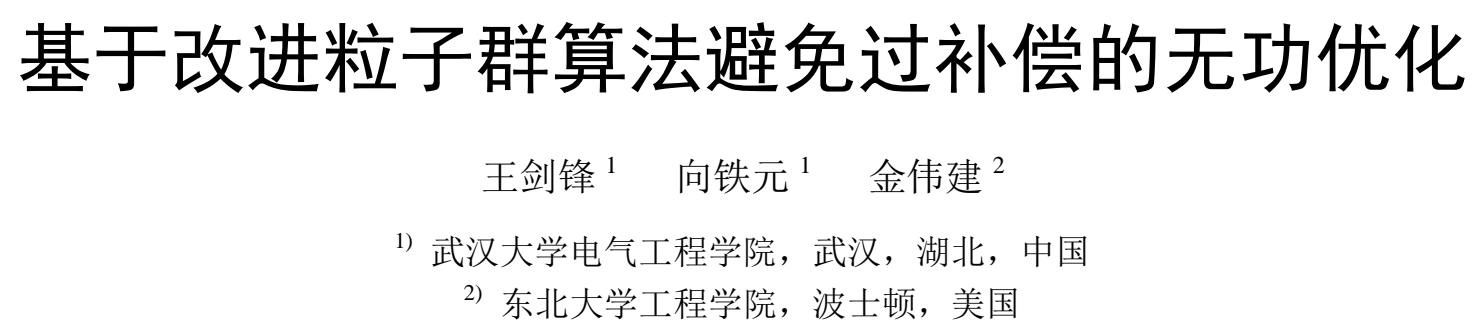

摘 要 将改进的粒子群算法应用于电力系统无功优化问题的求解, 克服了传统粒子群算法收玫精度不高、易陷入局部最优的缺 点。同时在操作过程中, 提出了一种通过动态调整补偿容量上限值避免产生过补偿现象的计算方法。对 IEEE6 节点系统进行无功优化 计算, 并与基本粒子群算法进行了比较, 结果表明该算法求得的有功损耗和电压水平较原状态均有较大的改善。

关键词 无功优化, 改进粒子群算法, 过补偿

\section{1. 引言}

无功优化是一种多变量、多约束、大规模的混合非线 性组合优化问题, 其控制变量既有连续变量, 又有离散变 量。常规优化方法是从初始解出发, 按照一定的轨迹不断 搜索新解, 最终收玫于最优解[1]。

目前关于无功补偿优化方法的研究较多, 其中粒子群 算法[2](Particle Swarm Optimization, PSO)等现代优化方法 也得到了广泛的应用[3], 但是在约束条件的设置中均没有 设置避免过补偿的措施。本文提出了通过动态调整节点补 偿容量上限避免系统产生过补偿的方法[4], 从而大大减少 了不可行解的产生, 提高了计算效率。本文对 PSO 算法加 以改进, 并将改进的 PSO 算法用于电力系统无功优化。对
IEEE6 节点系统进行仿真计算, 结果表明, 本文提出的算 法在优化结果和收敛特性上均具有优势。

\section{2. 无功优化数学模型}

在电力系统的有功潮流调度已给定的情况下, 以系统 网损最小为目标, 无功优化问题的数学模型表示为

$$
\begin{aligned}
\min P_{L}= & \sum_{K \in N_{E}} P_{k L}= \\
& \sum_{i \in N_{B}, j \in N_{i}} G_{i j}\left(V_{i}^{2}+V_{j}^{2}-2 V_{i} V_{j} \cos \theta_{i j}\right)
\end{aligned}
$$

且必须满足: 


$$
\begin{aligned}
& P_{G i}=P_{D i}+V_{i} \sum_{j \in N_{i}} V_{j}\left(G_{i j} \cos \theta_{i j}+B_{i j} \sin \theta_{i j}\right) \\
& \left.i \in \mathbb{N}_{P V}, n\right\} \\
& \begin{array}{c}
Q_{G i}=Q_{D i}+V_{i} \sum_{j \in N_{i}} V_{j}\left(G_{i j} \sin \theta_{i j}-B_{i j} \cos \theta_{i j}\right) \\
\left.i \in N_{P V}, n\right\}
\end{array} \\
& Q_{G i}^{\min } \leq Q_{G i} \leq Q_{G i}^{\max } \quad \mathrm{i} \in\left\{\mathrm{N}_{\mathrm{PV}}, \mathrm{n}\right\} \\
& V_{i}^{\min } \leq V_{i} \leq V_{i}^{\max } \quad \mathrm{i} \in \mathrm{N}_{\mathrm{PQ}} \\
& T_{k}^{\mathrm{mi} \mathrm{n}} \leq T_{k} \leq T_{k}^{\mathrm{max}} \quad \mathrm{k} \in \mathrm{N}_{\mathrm{T}} \\
& Q_{C i}^{\mathrm{mi}} \leq Q_{C i} \leq Q_{C i}^{\mathrm{max}} \quad \mathrm{i} \in \mathrm{N}_{\mathrm{C}} \\
& V_{i}^{\min } \leq V_{i} \leq V_{i}^{\max } \quad \mathrm{i} \in \mathrm{N}_{\mathrm{PV}}
\end{aligned}
$$

式中

$P_{L}$ 为系统的有功损耗; $N_{E}$ 为网络所有支路的集合; $P_{K L}$ 为第 $k$ 条支路的有功功率损耗; $N_{B}$ 为系统中所有母线 的集合; $N_{i}$ 为与第 $i$ 条母线相连的所有母线的集合, 包括 第 $i$ 条母线; $G_{i j} 、 B_{i j}$ 分别为第 $i$ 条母线和第 $j$ 条母线之间 的传输电导、传输电纳; $V_{i}$ 为第 $i$ 条母线的电压幅值; $\theta_{i j}$ 为第 $i$ 条母线和第 $j$ 条母线电压的相位差; $P_{G i}, Q_{G i}$ 分别 为第 $i$ 条母线所带发电机发出的有功功率、无功功率; $P_{D i}, Q_{D i}$ 分别为第 $i$ 条母线上所带负荷所需的有功功率、无 功功率; $N_{P V}$ 为所有 $P V$ 型母线的集合; $N_{P Q}$ 为所有 $P Q$ 型母线的集合; $N_{T}$ 为所有变压器支路的集合; $T_{K}$ 为第 $k$ 台变压器的分接头位置; $Q_{C i}$ 为第 $i$ 条母线上的无功电源补 偿; $N_{c}$ 为可以进行无功电源补偿的母线的集合; $n$ 指平衡 节点。

式(2)和式(3)是潮流方程的约束, 式(4)及式(5)是状态变 量的约束, 式(6) 式(8)是控制变量自身的约束。状态变量 是控制变量的函数, 隐含在潮流方程中。

本文用外点法构造辅助函数, 将状态变量约束以罚函 数 ${ }^{[5]}$ 形式计入目标函数中:

$$
\begin{array}{r}
\min F=P_{L}+\sum_{i \in N_{P Q}} \lambda_{1}\left(V_{i}-V_{i l i n}\right)^{2}+ \\
\sum_{i \in N_{P V}} \lambda_{2}\left(Q_{G i}-Q_{G i l i n}\right)^{2}
\end{array}
$$

其中: 等式第一项为电网的有功功率损耗, $N_{P Q}$ 和 $N_{P V}$ 分 别为 $P Q$ 节点和 $P V$ 节点的集合, $\lambda_{1}$ 和 $\lambda_{2}$ 为罚因子, $V_{i l i n}$ 和 $Q_{\text {Gilin }}$ 的定义如下:

$$
\begin{gathered}
V_{\text {ilin }}= \begin{cases}V_{i}^{\max } & V_{i}>V_{i}^{\max } \\
V_{i}^{\min } & V_{i}<V_{i}^{\min } \\
V_{i} & V_{i}^{\min } \leq V_{i} \leq V_{i}^{\max }\end{cases} \\
Q_{\text {Gilin }}= \begin{cases}Q_{i}^{\max } & Q_{i}>Q_{i}^{\max } \\
Q_{i}^{\min } & Q_{i}<Q_{i}^{\min } \\
Q_{i} & Q_{i}^{\min } \leq Q_{i} \leq Q_{i}^{\max }\end{cases}
\end{gathered}
$$

\section{3. 粒子群算法及改进}

\section{1 基本粒子群算法}

假设在一个 $D$ 维的搜索空间中, 有 $m$ 个粒子组成一个 群, 其中第 $i$ 个粒子在 $D$ 维搜索空间中的位置表示为向量 $x_{i}=\left(x_{i 1}, x_{i 2}, \cdots, x_{i D}\right)^{T}$, 其中 “飞翔”速度表示为 $\left.{ }_{i}=k_{i 1}, v_{i 2}{ } \cdots,{ }_{i} B\right), i=1,2, \cdots m$ 。每个粒子的位置代表了 待优化问题的一个可能的解, 它在空间中飞翔的轨迹代表 了搜索的过程。选取待优化问题的目标函数, 将 $x_{i}$ 代入目 标函数进行计算, 根据目标函数值的大小就可以衡量粒子 的优劣。对于最小化问题, 目标函数值越小的粒子其特性 越优。记第 $i$ 个粒子迄今为止搜索到的最优位置为 $p_{i}$, 称 为个体极值, 整个粒子群迄今为止搜索到的最优位置为 $p_{g}$, 称为全局极值。各粒子根据如下公式进行迭代操作, 从而更新自己的速度和位置 ${ }^{[4]}$ :

$$
\begin{gathered}
v_{i d}^{n+1}=\omega v_{i d}^{n}+c r_{1}^{n}\left(x_{i d}^{n}-x_{i d}^{n}\right)+c r_{2}^{n}\left(p_{g d}^{n}-x_{i d}^{n}\right) \\
x_{i d}^{n+1}=x_{i d}^{n}+v_{i d}^{n+1}
\end{gathered}
$$

式中: $d$ 表示 $D$ 维搜索空间中的第 $d$ 个变量, $d=1,2, \cdots, D ; c_{1}$ 和 $c_{2}$ 为非负加速度常速; $r_{1}$ 和 $r_{2}$ 是 $[0,1]$ 之间的随机数; $n$ 为迭代次数。 $\omega$ 是非负数, 称为惯性权 重。 $\omega$ 控制粒子的先前速度对当前速度的影响程度, 调整 算法全局和局部搜索能力的平衡。迭代终止条件根据具体 问题一般选为最大迭代次数或粒子群迄今为止搜索到的最 优解的目标函数值小于某一预定值。

\section{2 粒子群算法的改进}

针对粒子群算法存在的搜索精度不高、易陷于局部最 优解的缺点, 提出了一种改进的粒子群算法。

研究表明 ${ }^{[6]}$, 惯性因子 $\omega$ 对算法的优化性能有很大的 影响。较大的 $\omega$ 值有利于提高算法的收玫速度, 而 $\omega$ 较小 时则有利于提高算法的收敛精度。据此提出一种按式 (14) 
对 $\omega$ 进行自适应调整的策略, 即随着迭代的进行, 逐步减 少 $\omega$ 值。

$$
\omega(k)=\left[2 /\left(1+e^{\sigma k / k_{\max }}\right)\right] \omega^{\circ}
$$

式中: $\sigma$ 为正系数, 以调节 $\omega$ 的变化速度; $k_{\text {max }}$ 为迭代 次数的上限; $\omega^{\circ}$ 为 $\omega(k)$ 的上限; $k$ 为当前迭代次数。 对基本粒子群算法中式 (12) 的分析表明, 式中第 1 项 即 $\omega v_{i}(k)$ 体现了粒子的飞行惯性。采用固定的 $\omega$ 值时, 粒子的全局搜索能力和局部精细搜索能力的平衡性较 差, 因而在算法运行初期, 粒子能较快地飞向当前的全 局最优点, 但是当靠近全局最优时又往往会因为固定不 变的飞行惯性而不能对最优点进行精细搜索, 从而使得 收敛精度不高。本文的改进粒子群算法采用随迭代次数 逐步减小的 $\omega$ 值, 能灵活地调整粒子在全局和局部搜索 能力之间的平衡, 从而既可在初期有较高的收玫速度, 又可在后期有较高的收敛精度。

\section{4. 改进 PSO 求解无功优化}

\section{1 离散变量的处理}

在实际电力系统中, 节点电压可以连续变化, 但是变 压器的分接头位置和无功源的注入无功量一般按一定的步 长改变, 所以无功优化问题中既包含连续变量, 又包含离 散的整数控制变量。PSO 处理混合整数优化问题的一般方 法是将速度和位置归整为相近的整数, 本文通过映射编码 和取整的方法对离散变量进行处理。如对于一个变比在 $\left[T_{i}^{\mathrm{min}}, T_{i}^{\mathrm{max}}\right]$ 之间、调节步长为 $T_{\text {step }}$ 、共有 $l$ 档分接头的变 压器, 假设其对应第 $i$ 维控制变量 $x_{i}$, 则令 $x_{i}$ 的取值范围 等于分接头的档数, 即 $1 \leq x_{i} \leq l$, 粒子在该范围内初始化, 速度和位置更新后, 按式 (15) 将 $x_{i}$ 转化为相应的变比值 代入目标函数进行计算 ([ ] 表示取整):

$$
T_{i}=T_{i}^{\min }+\left[x_{i}-1\right] \times T_{\text {step }}
$$

\section{2 无功上限的动态调整}

现有的无功补偿优化方法在算法开始时设置了各节点 补偿容量的上限值, 这样无法避免在计算过程中产生过补 偿。当在某一节点设置补偿后, 从电源节点到达该节点线 路上的无功潮流已发生变化, 如果再根据原取值范围进行 操作可能造成过补偿。

实际应用中无法在保证最优补偿容量及位置的情况下 预先给定各节点不产生过补偿的补偿上限值。本文提出了 根据电网的无功潮流动态改变节点补偿容量上限的方法。
本文采用文献[5]中提出的方法, 形成一关联矩阵 $E$, 其含义为: 当节点 $i$ 的负荷功率流经线路 $j$ 时 $E(i, j)=1$; 否则 $E(i, j)=0$ 。利用矩阵 $E$ 可以方便地确定各补偿点的 补偿上限。例如要在节点 $k$ 处安装电容器, 则 $Q_{c k}^{\max }$ 确定方 法为:

$$
\begin{aligned}
Q_{c k}^{\max } & =\min _{i \in I_{k}} Q_{i} \\
I_{k} & =\{j \mid E(k, j)=1\}
\end{aligned}
$$

式中: $Q_{i}$ 为各线路中的无功潮流; $I_{k}$ 为从电源节点出 发到达节点 $k$ 的各线路集合。

\section{4. 算例分析}

本文分别用基本 PSO 和改进的 PSO 算法采用 Matlab 语言编写程序, 对 IEEE6 节点系统进行了无功优化计算, 并将结果与其初始结果进行了比较。

$I E E$ E 节点电力系统的网络参数见文献[6], 图 1 为 该系统接线图, 该系统包含 2 台发电机, 2 台可调变压器 和 2 个无功补偿负荷节点, 系统参数的基准容量为 $100 M V \bullet A$, 控制变量为发电机端电压 $V_{G 1} 、 V_{G 2}$, 变压 器分接头档位 $T_{65} 、 T_{34}$ 和电容器投切容量 $Q_{C 4} 、 Q_{C 6}$ 。其 中, 发电机 $G_{1} 、 G_{2}$ 端电压取值范围分别为 $(1.0 \sim 1.1)$ 和 $(1.1 \sim 1.15)$, 变压器档位调节范围为 $0.9 \sim 1.1$ 分为 8 个 档位, 单位档位调节量为 0.025 。电容器分 10 组投切, 节 点 4 和节点 6 处单位电容器容量分别为 $0.5 M V \bullet A$ 和 $0.55 M V \bullet A$ 。在系统初始状态下, 网络损耗为 $11.62 M W$, 节点 3 电压最低, 标么值为 0.8552 。

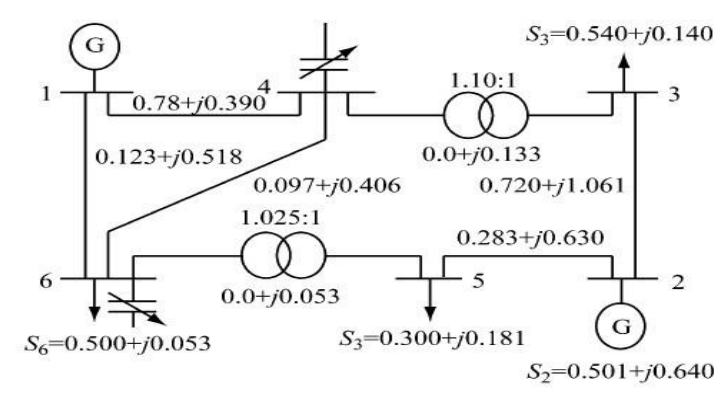

图 1 IEEE6 节点系统图

采用基本 PSO 和改进 PSO 算法对该系统进行无功优化 计算, 算法参数分别选为: 群体规模 $m=10, w=0.9$, $C_{1}=2, C_{2}=2, k_{\text {max }}=20$ 和群体规模 $m=10$, $w^{\circ}=0.9, C_{1}=2, C_{2}=2, k_{\max }=20, \sigma=1.3$ 。

图 2 为基本 PSO 和改进 PSO 避免无功过补偿算法的收玫过 
程曲线图。表 1 列出了采用基本 PSO、改进 PSO 和改进 PSO 避免无功过补偿算法得到的结果与该系统初始状态的 对比。

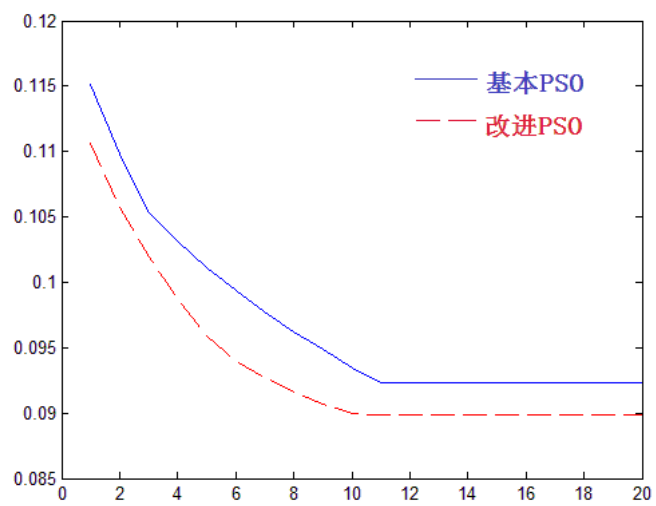

图 2 IEEE6 节点系统收敛过程曲线图

表 1 IEEE6 节点系统无功优化计算比较

\begin{tabular}{|c|c|c|c|c|}
\hline 节点电压 & 初始 & $\begin{array}{c}\text { 基本 } \\
\text { PSO }\end{array}$ & $\begin{array}{c}\text { 改进 } \\
\text { PSO }\end{array}$ & $\begin{array}{c}\text { 改进 PSO 避免 } \\
\text { 无功过补偿 }\end{array}$ \\
\hline 1 & 1.05 & 1.1 & 1.1 & 1.1 \\
\hline 2 & 1.1 & 1.15 & 1.15 & 1.15 \\
\hline 3 & 0.8552 & 1.0207 & 0.9996 & 1.028 \\
\hline 4 & 0.9525 & 0.9871 & 0.9911 & 0.9946 \\
\hline 5 & 0.9009 & 1.0104 & 1.0332 & 1.0161 \\
\hline 6 & 0.9332 & 0.9638 & 0.9655 & 0.9713 \\
\hline 有功损耗 & 0.1162 & 0.0908 & 0.0898 & 0.0884 \\
\hline
\end{tabular}

由图 2 可以看出, 改进 PSO 算法无论是在收玫速度还 是在收敛精度方面都较基本 PSO 算法有一定提高。由表 1 看出, IEEE6 节点系统用本文提出的改进 PSO 算法和改进 PSO 避免无功过补偿算法在满足各种约束条件的前提下, 均能有效降低有功损耗, 综合比较来看改进 PSO 避免过补 偿算法有明显优势, 其结果较初始状态系统有功损耗降低
了 $23.92 \%$, 负荷节点的最低电压由初始状态的 0.8552 升高 到优化后 0.9713 的, 可见电压质量明显提高。

\section{5. 结论}

本文提出了基于改进粒子群避免过补偿的无功优化算 法, 通过引入惯性变量增强了算法的收玫性和精确性, 通 过动态改变无功补偿上限的方法减少了大量的不可行解避 免了系统过补偿的发生。通过对 IEEE6 节点系统的计算表 明, 将该算法应用于电力系统无功优化是正确有效的, 具 有较稳定的收玫性能, 较高的计算精度, 同时也避免了系 统过补偿的发生。

\section{参考文献(References)}

[1] Su Ling, Zhao Dongmei and Han Jing, "Summarizaion of Reactive Power Optimization Algorithm in Electric Power System," Modern Electric Power, vol.21, no.6, pp.40-45, December 2004.

[2] Yuan Xiaohui, et al, "A Suvery on Application of Particle Swarm Optimization to Electric Power System," Power System Technology, vol.28, no.19, pp.14-18, October 2004.

[3] Yu Junxia, and Zhao Bo, "Improved Particle Swam Optimization Algorithm for Optimal Power Flow Problems," Proceedings of the CSU-EPSA, vol.17, no.4, pp.83-88, August 2005.

[4] Yao Zhuxiang, et al, "Model Aad Algorithm for Reactive Power Optimization with Penalty," Proceedings of the EPSA, vol.10, no.3, pp.39-45, September 1998.

[5] Yang Jianjun, “Application of Improved Genetic Algorithm for Avoiding over Compensation in the Optimization of Centralized Reactive Power Compensation," Electrotechnical Application, vol.24, no.10, pp.35-39, 2005.

[6] Shi Y, and Eberhart R, "A Modified Particle Swarm Optimizer," IEEE World Congress on Computational Intelligence, pp.69-73, 1998. 